\title{
Significance of Wave-Particle Interaction Analyzer for direct measurements of nonlinear wave-particle interactions
}

\author{
Y. Katoh ${ }^{1}$, M. Kitahara ${ }^{1}$, H. Kojima ${ }^{2}$, Y. Omura ${ }^{2}$, S. Kasahara ${ }^{3}$, M. Hirahara ${ }^{4}$, Y. Miyoshi ${ }^{4}$, K. Seki ${ }^{4}$, K. Asamura ${ }^{3}$, \\ T. Takashima ${ }^{3}$, and T. Ono ${ }^{1}$ \\ ${ }^{1}$ Department of Geophysics, Graduate School of Science, Tohoku University, Miyagi 980-8578, Japan \\ ${ }^{2}$ Research Institute for Sustainable Humanosphere, Kyoto University, Kyoto 611-0011, Japan \\ ${ }^{3}$ ISAS, JAXA, Kanagawa 229-8510, Japan \\ ${ }^{4}$ Solar-Terrestrial Environment Laboratory, Nagoya University, Aichi 464-8601, Japan
}

Correspondence to: Y. Katoh (yuto@stpp.gp.tohoku.ac.jp)

Received: 26 October 2012 - Revised: 24 January 2013 - Accepted: 18 February 2013 - Published: 19 March 2013

\begin{abstract}
In the upcoming JAXA/ERG satellite mission, Wave Particle Interaction Analyzer (WPIA) will be installed as an onboard software function. We study the statistical significance of the WPIA for measurement of the energy transfer process between energetic electrons and whistler-mode chorus emissions in the Earth's inner magnetosphere. The WPIA measures a relative phase angle between the wave vector $\boldsymbol{E}$ and velocity vector $\boldsymbol{v}$ of each electron and computes their inner product $W$, where $W$ is the time variation of the kinetic energy of energetic electrons interacting with plasma waves. We evaluate the feasibility by applying the WPIA analysis to the simulation results of whistler-mode chorus generation. We compute $W$ using both a wave electric field vector observed at a fixed point in the simulation system and a velocity vector of each energetic electron passing through this point. By summing up $W_{i}$ of an individual particle $i$ to give $W_{\text {int }}$, we obtain significant values of $W_{\text {int }}$ as expected from the evolution of chorus emissions in the simulation result. We can discuss the efficiency of the energy exchange through wave-particle interactions by selecting the range of the kinetic energy and pitch angle of the electrons used in the computation of $W_{\text {int }}$. The statistical significance of the obtained $W_{\text {int }}$ is evaluated by calculating the standard deviation $\sigma_{W}$ of $W_{\text {int }}$. In the results of the analysis, positive or negative $W_{\text {int }}$ is obtained at the different regions of velocity phase space, while at the specific regions the obtained $W_{\text {int }}$ values are significantly greater than $\sigma_{W}$, indicating efficient wave-particle interactions. The present study demonstrates the feasibility of using the WPIA, which will be on
\end{abstract}

board the upcoming ERG satellite, for direct measurement of wave-particle interactions.

Keywords. Magnetospheric physics (Plasma waves and instabilities) - Space plasma physics (Wave-particle interactions; Instruments and techniques)

\section{Introduction}

Wave-particle interactions in space plasmas occur within time scales characteristic of plasma particles, such as the gyroperiod and plasma oscillation period. These time scales are, especially for electrons in the magnetosphere, too short to detect wave-particle interactions in situ using conventional plasma instruments. As an example, we focus on cyclotron resonant interactions of energetic electrons with a coherent whistler-mode wave in the magnetosphere. The time scale associated with the motion of resonant electrons nonlinearly trapped by a coherent whistler-mode wave is characterised by the oscillation period of electrons within the trapping region in velocity phase space (e.g., Matsumoto, 1985), and is one or two orders of magnitude longer than the gyroperiod of electrons. The trapping oscillations of resonant electrons occur within a limited region of velocity phase space determined by the cyclotron resonance condition and within a certain relative phase range with respect to the wave magnetic field vector. Oscillations of resonant electrons result in a relaxation of the velocity distribution within the trapping region and saturation of the interactions within time periods of hundreds or thousands of electron gyroperiods (e.g., 
Katoh and Omura, 2004) corresponding to tens of milliseconds. Using plasma instruments on board spacecraft, which mostly have time resolutions of a few tens of milliseconds or less, it is impossible to directly measure relaxation of the electron velocity distribution or energy exchange processes during wave-electron interactions.

One observation method that enables wave-particle interactions to be investigated is a comparison of the observed wave phase and particles. There have been many attempts to measure interactions between electron beams and electrostatic waves, while previous instruments counted the number of particles by referring the observed wave phase and measured the electron distribution in the wave phase space (Ergun et al., 1991, 1998; Gough et al., 1995; Buckley et al., 2000). Recently Fukuhara et al. (2009) proposed a new type of instrument for direct and quantitative measurements of wave-particle interactions, which is referred to as "WaveParticle Interaction Analyzer (WPIA)." The WPIA uses all of the three-dimensional properties of the observed waveforms and particles and quantifies the kinetic energy flow by measuring the inner product of the observed instantaneous wave and the particle velocity vectors. To apply the WPIA to space plasma measurement, it is necessary to obtain both the wave field vector and the velocity of each incoming particle on a time scale sufficient to resolve variations in the wave phase and the gyro-motion of particles. Since the sampling clock in wave and plasma instruments can be adjusted to the order of microseconds, measurement of the relative phase can be carried out using state-of-the-art instruments. It is not necessary for all of waveform and particle data to be transferred to the ground in order to apply the WPIA. Accumulation of the inner product can be conducted onboard, so that the telemetry data size can be optimised to a realistic size by sending only the results of the accumulation. In the upcoming JAXA satellite mission: Exploration of energization and Radiation in Geospace (ERG) project (Miyoshi et al., 2012), WPIA measurements will be carried out using Software-type WPIA (S-WPIA). One of the primary targets of this mission is to clarify the interaction of relativistic electrons with chorus emissions. During the mission, the onboard S-WPIA will be used to investigate whistler-mode wave-particle interactions for the first time.

Recently, the gyroresonant interaction of relativistic electrons with whistler-mode chorus emissions has been investigated as a possible generation mechanism for $\mathrm{MeV}$ electrons in the outer radiation belt (e.g., Shprits et al., 2008; Thorne, 2010; Ebihara and Miyoshi, 2011, for review). Observations have revealed the possible roles of chorus in controlling the flux of radiation belt electrons (e.g., Miyoshi et al., 2003, 2007; Horne et al., 2003; Kasahara et al., 2009). As theoretical and simulation studies have clarified, nonlinear waveparticle interactions are essential when considering the generation process of coherent chorus elements with rising tones (Nunn, 1974; Nunn et al., 1997; Trakhtengerts, 1995, 1999; Katoh and Omura, 2007a, 2011; Omura et al., 2008, 2009;
Hikishima et al., 2009; Omura and Nunn, 2011) and with falling tones (Nunn and Omura, 2012) and rapid energisation of relativistic electrons by chorus emissions (Katoh and Omura, 2007b; Omura et al., 2007; Summers and Omura, 2007; Furuya et al., 2008; Katoh et al., 2008). In particular, the formation of an electromagnetic electron hole in velocity phase space is a key process related to nonlinear interactions with chorus emissions. The electromagnetic electron hole is produced by depletion of velocity phase space due to nonlinear interactions between a coherent whistler-mode wave and resonant electrons moving along the field lines around the magnetic equator. The presence of the hole results in the formation of nonlinear resonant currents that contribute to the growth of coherent wave elements with a specific phase variation with significantly large growth rate rather than the linear growth rate. In addition, a fraction of the resonant electrons trapped in the hole undergo very efficient acceleration, while the majority which are outside the hole and are untrapped by wave elements lose their kinetic energy in generating chorus emissions. Based on the results of previous theoretical and simulation studies on chorus emissions, the nonlinear theory is promising and can be verified by obtaining evidence of an electromagnetic electron hole in the equatorial region of the inner magnetosphere during typical chorus events. However, the greatest difficulty with regard to direct measurements is that the hole is formed in a specific phase range relative to the wave magnetic field vector rotating with the wave frequency, which is several kilohertz in the inner magnetosphere. This is a crucial problem for conventional plasma instruments, but can be overcome by the WPIA.

In the WPIA, $W\left(t_{0}\right)=q \boldsymbol{E}\left(t_{0}\right) \cdot \boldsymbol{v}\left(t_{0}\right)$ is computed using the wave electric field vector measured by the plasma wave instrument and the velocity vector of an energetic electron detected by the particle instrument at $t=t_{0}$. Considering the time variation of the kinetic energy, we obtain

$\frac{d K}{d t}=m_{0} \boldsymbol{v} \times \frac{d(\gamma \boldsymbol{v})}{d t}=q \boldsymbol{E} \times \boldsymbol{v}=W$,

where $K=m_{0} c^{2}(\gamma-1)$ is the kinetic energy of a charged particle including relativistic effects, $m_{0}$ and $q$ are the rest mass and charge of a charged particle, respectively, $c$ is the speed of light, and $\gamma$ is the Lorentz factor given by $\gamma=$ $\left[1-(v / c)^{2}\right]^{-1 / 2}$. Since Eq. (1) indicates that $W$ represents the time variation of the kinetic energy of a charged particle due to wave-particle interactions, by summing up $W_{i}$ of an individual particle $i$ to give $W_{\text {int }}=\sum_{i=1}^{N} W_{i}$, we can measure the amount of energy transferred between waves and particles in a volume filled by $N$ plasma particles. In the presence of efficient wave-particle interactions, the value of $W_{\text {int }}$ can be either positive or negative corresponding to particle acceleration or wave generation, respectively. In practice, however, the limited data availability (e.g., finite particle count) 
restricts the accuracy of the calculations, so that the statistical significance of the obtained $W_{\text {int }}$ must be carefully evaluated.

In the present study, to evaluate the statistical significance of WPIA measurements of wave-particle interactions, the WPIA method is applied to dataset of the simulation results reproducing whistler-mode chorus generation (e.g., Katoh and Omura, 2007a). The inner product $W$ is computed for a wave electric field observed at a fixed point in the simulation system and the velocity vector of each energetic electron passing through that point. We propose that the statistical significance can be evaluated based on the standard deviation $\sigma_{W}$ of $W_{\text {int }}$ and discuss the feasibility quantitatively by applying the method to the simulation result. The outline of this paper is as follows. The meaning of the quantity $W$ is discussed in Sect. 2. In Sect. 3, we briefly describe the simulation model and results reproducing the chorus generation process. We show results of the WPIA analysis in Sect. 4 and discuss the statistical significance in Sect. 5. Section 6 gives summary of the present study.

\section{WPIA}

We examine the meaning of $W$ in detail. In the present study, ions are assumed to be an immobile neutralising component. We assume that the current density $\boldsymbol{J}$ consists of a cold plasma component $\boldsymbol{J}_{\mathrm{c}}$ and a hot component $\boldsymbol{J}_{\mathrm{R}}$, namely, $\boldsymbol{J}=$ $\boldsymbol{J}_{\mathrm{c}}+\boldsymbol{J}_{\mathrm{R}}$. The cold component $\boldsymbol{J}_{\mathrm{c}}$ is given by $\boldsymbol{J}_{\mathrm{c}}=-e N_{\mathrm{c}} \boldsymbol{u}_{\mathrm{c}}$, where $N_{\mathrm{c}}$ and $\boldsymbol{u}_{\mathrm{c}}$ are the number density and velocity of the cold plasma component, respectively. By taking the inner product of $\boldsymbol{E}$ and $\boldsymbol{J}$, we obtain

$\boldsymbol{J} \cdot \boldsymbol{E}=\boldsymbol{J}_{\mathrm{c}} \cdot \boldsymbol{E}+\boldsymbol{J}_{R} \cdot \boldsymbol{E}$.

The equation of the motion of the cold plasma component is given by

$$
\frac{\partial \boldsymbol{u}_{c}}{\partial t}=-\frac{e}{m_{\mathrm{e}}}\left(\boldsymbol{E}+\boldsymbol{u}_{\mathrm{c}} \times \boldsymbol{B}_{0}\right),
$$

where $m_{\mathrm{e}}$ is the mass of an electron and $\boldsymbol{B}_{0}$ is the background magnetic field. By taking the inner product of $\boldsymbol{u}_{\mathrm{c}}$ with both sides of Eq. (3), we obtain

$\boldsymbol{u}_{\mathrm{c}} \cdot \frac{\partial \boldsymbol{u}_{\mathrm{c}}}{\partial t}=-\frac{e}{m_{\mathrm{e}}} \boldsymbol{u}_{\mathrm{c}} \cdot \boldsymbol{E}$.

Using Eq. (4), we find that the time variation of the kinetic energy of the cold plasma component is given by

$$
\frac{\partial}{\partial t}\left(\frac{1}{2} N_{\mathrm{c}} m_{\mathrm{e}} \boldsymbol{u}_{\mathrm{c}}^{2}\right)=N_{\mathrm{c}} m_{\mathrm{e}} \boldsymbol{u}_{\mathrm{c}} \cdot \frac{\partial \boldsymbol{u}_{\mathrm{c}}}{\partial t}=\boldsymbol{J}_{\mathrm{c}} \cdot \boldsymbol{E} .
$$

From the divergence of the Poynting vector $S$, we obtain

$\nabla \cdot S=\frac{\partial}{\partial t}\left(\frac{\varepsilon_{0}}{2} \boldsymbol{E}^{2}+\frac{1}{2 \mu_{0}} \boldsymbol{B}^{2}\right)+\boldsymbol{J} \cdot \boldsymbol{E}$, where $\varepsilon_{0}$ and $\mu_{0}$ are the permittivity and the magnetic permeability in vacuum, respectively. From Eqs. (2) and (5) we obtain

$\nabla \cdot S=\frac{\partial U}{\partial t}+\boldsymbol{J}_{\mathrm{R}} \cdot \boldsymbol{E}$,

where the variable $U$ is the energy density of the plasma waves and $U=\frac{\varepsilon_{0}}{2} \boldsymbol{E}^{2}+\frac{1}{2 \mu_{0}} \boldsymbol{B}^{2}+\frac{1}{2} N_{c} m_{\mathrm{e}} \boldsymbol{u}_{\mathrm{c}}^{2}$. Let us consider a wave generation region $V$, and integrate Eq. (7) over the volume $V$, given by

$\int_{V} \nabla \cdot \boldsymbol{S} d V=\frac{\partial}{\partial t} \int_{V} U d V+\int_{V} \boldsymbol{J}_{\mathrm{R}} \cdot \boldsymbol{E} d V$.

On the surface $A$ of the generation region $V$, we have

$\int_{A} \nabla \cdot \boldsymbol{S} d A=\frac{\partial}{\partial t} \int_{V} U d V+\int_{V} \boldsymbol{J}_{\mathrm{R}} \cdot \boldsymbol{E} d V$

and

$\int_{A} \nabla \cdot S d A>0$.

From Eqs. (9) and (10), we obtain

$\frac{\partial}{\partial t} \int_{V} U d V>-\int_{V} \boldsymbol{J}_{\mathrm{R}} \cdot \boldsymbol{E} d V$.

If $\int_{V} \boldsymbol{J}_{\mathrm{R}} \cdot \boldsymbol{E} d V<0$, we have

$\frac{\partial}{\partial t} \int_{V} U d V>0$

which suggests the plasma wave energy is growing in time, i.e., an absolute instability such as generation of chorus is taking place in the volume $V$.

As a proxy of $\int_{V} \boldsymbol{J}_{\mathrm{R}} \cdot \boldsymbol{E} d V$, we measure $\Delta W(\boldsymbol{r}, t)$ emerged during a time interval $\Delta t$, given by

$\Delta W(\boldsymbol{r}, t)=\int_{t}^{t+\Delta t} \boldsymbol{E}\left(\boldsymbol{r}, t^{\prime}\right) \cdot \boldsymbol{J}_{\mathrm{R}}\left(\boldsymbol{r}, t^{\prime}\right) \mathrm{d} t^{\prime}$.

The current density of the hot component $\boldsymbol{J}_{\mathrm{R}}$ is given by

$\boldsymbol{J}_{\mathrm{R}}(\boldsymbol{r}, t)=\iiint q \boldsymbol{v} f(\boldsymbol{r}, \boldsymbol{v}, t) \mathrm{d} \boldsymbol{v}$,

where $f$ is the phase space density of the hot plasma component. Substituting Eq. (14) into Eq. (13), we obtain

$\Delta W(\boldsymbol{r}, t)=\int_{t}^{t+\Delta t} \iiint q \boldsymbol{E}\left(\boldsymbol{r}, t^{\prime}\right) \cdot \boldsymbol{v} f\left(\boldsymbol{r}, \boldsymbol{v}, t^{\prime}\right) \mathrm{d} \boldsymbol{v} \mathrm{d} t^{\prime}$. 
(a) $W<0$

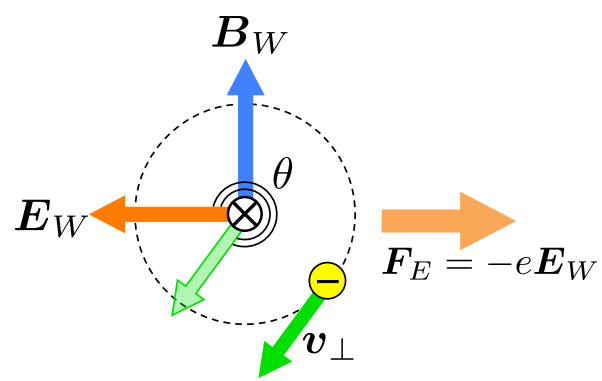

(b) $W>0$

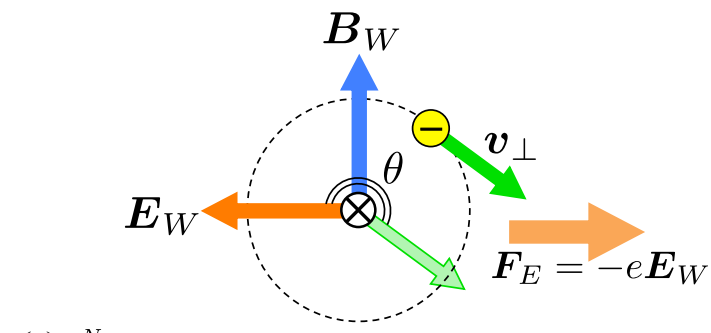

(c)

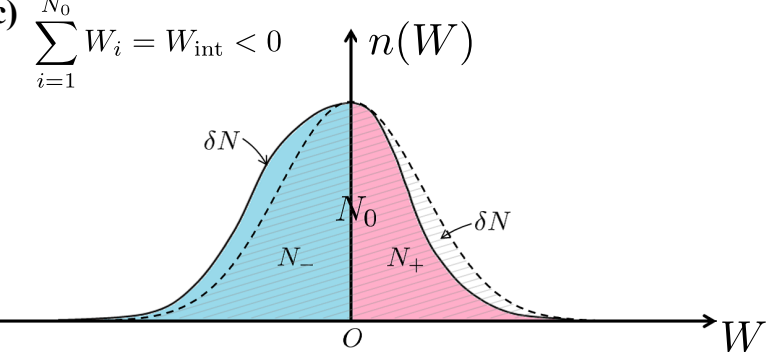

Fig. 1. Schematic illustration of the WPIA for whistler-mode waveparticle interactions. (a) and (b) represent the relation between the perpendicular component of the velocity of an energetic electron, and the wave electric $\left(\boldsymbol{E}_{W}\right)$ and magnetic $\left(\boldsymbol{B}_{W}\right)$ fields of whistlermode waves propagating parallel to the background magnetic field direction for the cases of (a) $W<0$ and (b) $W>0$. (c) Distribution of energetic electrons as a function of $W$ for an example of a negative $W_{\text {int }}$ corresponding to wave generation. The total number of energetic electrons is $N_{0}$, while $N_{+}$and $N_{-}$are the numbers of energetic electrons having positive and negative $W$, respectively.

Since measurement of the phase space density $f$ is conducted for discrete times, we re-express $\Delta W(\boldsymbol{r}, t)$ as a summation of $q \boldsymbol{E} \cdot \boldsymbol{v}$ measured over the time interval $\Delta t$, given by

$$
\Delta W(\boldsymbol{r}, t)=\sum_{t \leq t_{i} \leq t+\Delta t}^{N} q \boldsymbol{E}\left(\boldsymbol{r}, t_{i}\right) \cdot \boldsymbol{v}_{i},
$$

where $N$ denotes the number of particles detected during the $\Delta t$ interval, the subscript $i$ designates quantities of the $i$ th particle, and $t_{i}$ is the timing of the detection of the $i$-th particle. We note that $t_{i}$ can be identical for different $i$. We also note that the expression of $\Delta W$ given by Eq. (16) is an alternative representation of the quantity " $I$ " introduced by Fukuhara et al. (2009). To obtain $\Delta W$, we should determine the time interval $\Delta t$ of the accumulation from the time-scale of wave-particle interactions. The time resolution of $t_{i}$ should be shorter than the time-scale of wave-particle interactions, but $\Delta t$ should be sufficiently longer than the time-scale of wave-particle interactions to observe energy exchange between waves and particles. In addition, since we use a waveform and a finite number of particles obtained during a finite time interval, statistical fluctuation should be considered in the measurement of this energy exchange.

Figure 1 shows a schematic illustration of the WPIA for interactions between energetic electrons and whistler-mode waves propagating parallel to the background magnetic field direction. Figure $1 \mathrm{a}$ and $\mathrm{b}$ show the relation between the perpendicular component of the velocity of an energetic electron, and the wave electric $\left(\boldsymbol{E}_{W}\right)$ and magnetic fields $\left(\boldsymbol{B}_{W}\right)$ of whistler-mode waves for the cases of $W<0$ and $W>0$. The sign of $W$ is determined by the relative angle $\theta$ between $\boldsymbol{v}_{\perp}$ and $\boldsymbol{E}_{W}$. Here we assume that a finite volume is filled by $N_{0}$ plasma particles and that a fraction of these particles resonate with whistler-mode waves propagating in the volume. Since we can evaluate the time variation of the kinetic energy $W_{i}$ of each particle, the net energy exchange between the particles and waves in the volume can be evaluated by accumulating $W_{i}$ over all $N_{0}$ particles to obtain $W_{\text {int }}$. Figure 1c shows a distribution of energetic electrons in the volume as a function of $W$ for an example with a negative $W_{\text {int }}$, which corresponds to wave generation. We denote the numbers of energetic electrons having positive and negative $W$ as $N_{+}$ and $N_{-}$, respectively. If efficient energy exchange occurs in the volume, then the net energy exchange originates in energetic electrons satisfying the cyclotron resonance condition with whistler-mode waves. In such a case, $N_{+}$and $N_{-}$should be significantly different from each other, while similar $N_{+}$ and $N_{-}$would be obtained if only non-resonant electrons were present. Figure 1c corresponds to the case of efficient wave-particle interactions resulting in the wave generation, where $N_{-}$is larger than $N_{+}$to satisfy that $W_{\text {int }}$ is negative. As shown, the difference $\delta N$ between $N_{+}$and $N_{-}$makes the distribution $n(W)$ asymmetric. If $\delta N$ was negligible and the resultant $W_{\text {int }}$ was almost zero, essentially no net energy exchange would occur: i.e., the waves would be merely propagating in the volume without growing nor damping.

Since the number of particles in the volume is finite, there must be fluctuation of $W_{\text {int }}$ over time. This fluctuation originates from thermal fluctuation of energetic electrons and fluctuation of both wave electric field amplitude and relative angle $\theta$. The value of $W_{\text {int }}$ should be sufficiently larger than the fluctuation, particularly for the case of efficient waveparticle interactions. Based on the central limit theorem, we can evaluate the statistical significance of the obtained $W_{\text {int }}$ by computing the standard deviation $\sigma_{W}$. Here we consider the integration of $W(t)$ over a time interval $d T$ for the case of $N$ energetic particles. In this case, we can evaluate $\sigma_{W}$ of 
the obtained $W_{\text {int }}$ by computing

$\sigma_{W}=\sqrt{\sum_{i=1}^{N}\left(q \boldsymbol{E}_{W}\left(t_{i}\right) \cdot \boldsymbol{v}_{i}\right)^{2}-\frac{1}{N}\left(\sum_{i=1}^{N} q \boldsymbol{E}_{W}\left(t_{i}\right) \cdot \boldsymbol{v}_{i}\right)^{2}}$.

Here the first and second terms on the right-hand side of Eq. (17) correspond to the width and the centre of the distribution of $q \boldsymbol{E}_{W}(t) \cdot \boldsymbol{v}$, respectively. In the WPIA, we can identify the energy exchange between waves and particles if we can obtain a $W_{\text {int }}$ sufficiently larger than $\sigma_{W}$. The integration of $W(t)$ over time should be conducted until the obtained $W_{\text {int }}$ exceeds the value of $n \sigma_{W}$ corresponding to the required statistical significance: assuming a Gaussian distribution, $n=1.64$ for a statistical significance of $90 \%$ and $n=1.96$ for $95 \%$.

\section{Simulation of chorus generation}

To evaluate the feasibility of the WPIA method, we analyse simulation results reproducing chorus emissions. We use an electron hybrid code with a dipole magnetic field (Katoh and Omura, 2006, 2007a, b, 2011; Omura et al., 2008), in which cold electrons are treated as a fluid and energetic electrons as particles using the particle-in-cell (PIC) method including fully relativistic effect. We assume a spatially onedimensional system along the background magnetic field direction, with $h$ as the distance from the magnetic equator. Although the simulation system is one-dimensional, we take into account the mirror motion of energetic electrons in the dipole magnetic field by using a cylindrical field model (Katoh and Omura, 2006). We solve Maxwell's equations with the current densities computed from the motion of electrons of both cold and energetic components. We treat transverse electromagnetic waves propagating parallel to the dipole magnetic field, while we neglect the longitudinal component of the electric field.

The time step and the grid spacing of the simulation are $0.01 \Omega_{\mathrm{e} 0}^{-1}$ and $0.06 c \Omega_{\mathrm{e} 0}^{-1}$, respectively, where $\Omega_{\mathrm{e} 0}$ is the electron gyrofrequency at the magnetic equator. The simulation system is along the field line and the number of grid points $L_{h}=16384$. The number of particles initially placed in the simulation system representing energetic electrons is $4096 \times L_{h}$. We assume that the plasma frequency of the cold electrons $\omega_{\text {pe }}$ at the equator is $4 \Omega_{\mathrm{e} 0}$, while the number density of cold electrons $N_{\mathrm{e}, \text { cold }}$ is constant in space and time. Because of limited computational resources, we assume that the spatial inhomogeneity of the background magnetic field is larger than the realistic dipole field of $L=4$ of the Earth's magnetosphere. Approximating the spatial inhomogeneity of the dipole magnetic field in the equatorial region of the magnetosphere as $\Omega_{\mathrm{e}}(h)=\Omega_{\mathrm{e} 0}\left(1+a h^{2}\right)$, the constant $a$ is given by $4.5 /\left(L R_{\mathrm{E}}\right)^{2}$ for the Earth's magnetosphere, while we assume $4.5\left(3.5 / L R_{\mathrm{E}}\right)^{2}=55 /\left(L R_{\mathrm{E}}\right)^{2}$ in the simulation.
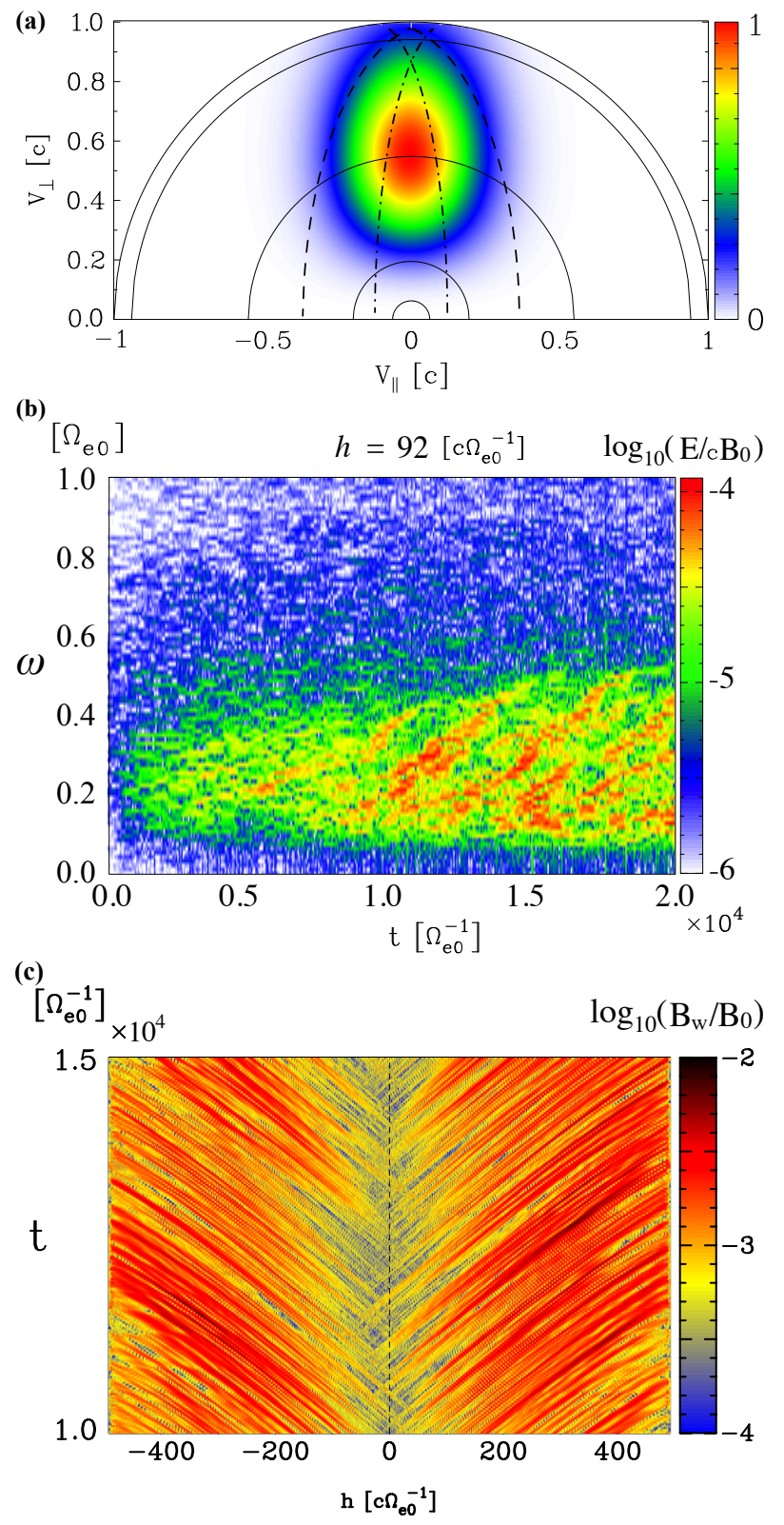

Fig. 2. Simulation results of the electron hybrid code used for the pseudo-observations of the WPIA. (a) The initial velocity distribution of energetic electrons assumed at the magnetic equator. Dashed and dash-dotted lines respectively denote resonance ellipses of $\omega=0.2$ and $0.5 \Omega_{\mathrm{e} 0}$ (after Summers et al., 1998). The five solid semicircles represent constant energy contours of $1 \mathrm{keV}, 10 \mathrm{keV}$, $100 \mathrm{keV}$, and $1 \mathrm{MeV}$, and the speed of light. (b) Spectrogram observed at the fixed point $h=92 c \Omega_{\mathrm{e} 0}^{-1}$ in the Northern Hemisphere of the simulation system. (c) Time evolution of the spatial profile of the wave magnetic field amplitude.

We assume an anisotropic velocity distribution with a loss cone for the initial velocity distribution $f\left(v_{\|}, v_{\perp}\right)$ of energetic electrons at the magnetic equator, given by 


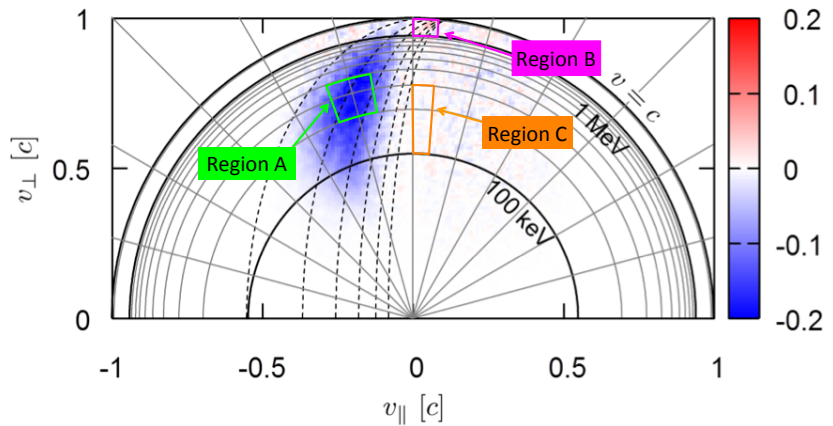

Fig. 3. Distribution of $W_{\text {int }}$ in velocity space computed at $h=$ $+200 c \Omega_{\mathrm{e} 0}^{-1}$ over the time interval from 0 to $20000 \Omega_{\mathrm{e} 0}^{-1}$. Dashed lines denote resonance ellipses of forward propagating whistlermode waves of $\omega=0.1,0.2,0.3,0.4,0.5$, and $0.6 \Omega_{\mathrm{e} 0}$ (after Summers et al., 1998). Coloured rectangles represent the energy and pitch angle ranges of Regions $\mathrm{A}, \mathrm{B}$, and $\mathrm{C}$ given in Table 1 .

$f\left(v_{\|}, v_{\perp}\right)=A_{0} \exp \left(-\frac{v_{\|}^{2}}{2 V_{t \|}^{2}}\right) g\left(v_{\perp}\right)$

and

$g\left(v_{\perp}\right)=\frac{1}{1-\beta}\left\{\exp \left(-\frac{v_{\perp}^{2}}{2 V_{t \perp}^{2}}\right)-\exp \left(-\frac{v_{\perp}^{2}}{2 \beta V_{t \perp}^{2}}\right)\right\}$,

where $A_{0}$ is a constant, $\beta=0.5$, and $V_{t \|}$ and $V_{t \perp}$ are thermal velocities of energetic electrons in the directions parallel and perpendicular to the background magnetic field, respectively. We assume that the thermal velocities $V_{t \|}$ and $V_{t \perp}$ are $0.225 c$ and $0.6 c$, respectively, and that the perpendicular component of the effective thermal velocity $\left\langle V_{t \perp}\right\rangle$ is $\sqrt{2(1+\beta)} V_{t \perp}$. The anisotropic velocity distribution of energetic electrons drives a linear instability generating a band of whistler-mode waves.

Figure 2 summarises the simulation results reproducing the generation process of chorus emissions with rising tones (Katoh and Omura, 2007a). In the early stage of the simulation $\left(t=0-2500 \Omega_{\mathrm{e} 0}^{-1}\right)$, a band of whistler-mode waves is generated through the instability driven by the temperature anisotropy of energetic electrons. The time scale and the frequency range of the evolution of whistler-mode waves are consistent with the growth rate predicted by the linear theory including the relativistic effect (Xiao et al., 1998; Katoh and Omura, 2011). As shown in Fig. 2a, after $t=2500 \Omega_{\mathrm{e} 0}^{-1}$, we observe the generation of coherent wave elements with rising tones. Details of the generation process reproduced in the simulation results have been discussed in previous studies (Katoh and Omura, 2007a, 2011; Omrua et al., 2008, 2009; Omura and Nunn, 2011). Coherent wave elements are successively generated from the magnetic equator, while the wave intensity of each element is significantly amplified during its propagation away from the equator, as shown in Fig. $2 b$.
Table 1. Definition of Regions A, B, and C.

\begin{tabular}{lccc}
\hline & Energy range & $\begin{array}{c}\text { Pitch angle } \\
\text { range [deg.] }\end{array}$ & $\begin{array}{c}\text { Sign of } \\
W_{\text {int }}\end{array}$ \\
\hline Region A & $200-400 \mathrm{keV}$ & $100-110$ & negative \\
Region B & $1-4 \mathrm{MeV}$ & $85-90$ & positive \\
Region C & $100-300 \mathrm{keV}$ & $85-90$ & - \\
\hline
\end{tabular}

\section{WPIA in the simulation results}

Using the simulation results for the chorus generation, we conduct a WPIA analysis. We compute $W(t)=q \boldsymbol{E}_{W}(t) \cdot \boldsymbol{v}(t)$ from the wave electric field $\boldsymbol{E}_{W}$ observed at a fixed point in the simulation system and the velocity vector $\boldsymbol{v}$ of each energetic electron passing through the observation point. The computed $W(t)$ is integrated over time to obtain $W_{\text {int. }}$. Figure 3 shows the distribution of the obtained $W_{\text {int }}$ in the velocity space at $h=+200 c \Omega_{\mathrm{e} 0}^{-1}$ for the time interval from 0 to $20000 \Omega_{\mathrm{e} 0}^{-1}$. Since chorus emissions generated from the magnetic equator propagate away from the equator, energetic electrons of tens or hundreds of $\mathrm{keV}$ moving toward the equator satisfy the cyclotron resonance condition; the resonant electrons have negative values of $v_{\|}$at $h=+200 c \Omega_{\mathrm{e} 0}^{-1}$. Figure 3 reveals that the kinetic energy of electrons having large pitch angles and $v_{\|}$opposite to the wave normal direction is transferred to waves in significant amount, resulting in the efficient wave growth as shown in Fig. 2b.

For the discussion of the statistical significance of $W_{\text {int }}$ obtained by simulation, we specify three regions in the velocity phase space as given in Table 1 and shown in Fig. 3. We evaluate $W_{\text {int }}$ and $\sigma_{W}$ separately for each region by using the energetic electrons in the corresponding kinetic energy and pitch angle ranges. Figure 4a shows the time history of $W_{\text {int }}$ computed in Region A of velocity space. We find that $W_{\text {int }}$ decreased over time, demonstrating the growth of chorus emissions. Using the dashed lines included in Fig. 4a, which show the values $W_{\text {int }} \pm 1.96 \sigma_{W}$, we can verify that the kinetic energy of the energetic electrons in Region A decreased to a statistically significant degree; this difference was transferred to plasma waves contributing the generation of chorus emissions. Figure $4 \mathrm{~b}$ shows the results computed for Region B. The value of $W_{\text {int }}$ increased over time and became larger than the values of $1.96 \sigma_{W}$ after $t=16000 \Omega_{\mathrm{e} 0}^{-1}$. Based on the results shown in Fig. 4b, we can verify with sufficient statistical certainty that relativistic electrons obtained kinetic energy from plasma waves in Region B. Finally, we analyse $W_{\text {int }}$ obtained in Region C. Since energetic electrons in Region $\mathrm{C}$ do not satisfy the cyclotron resonance condition with whistler-mode waves generated in the simulation result, we expect that efficient wave-particle interactions will not be found in Region C. Figure 4c shows that $W_{\text {int }}$ in Region $\mathrm{C}$ fluctuated over time, but not significantly relative to 

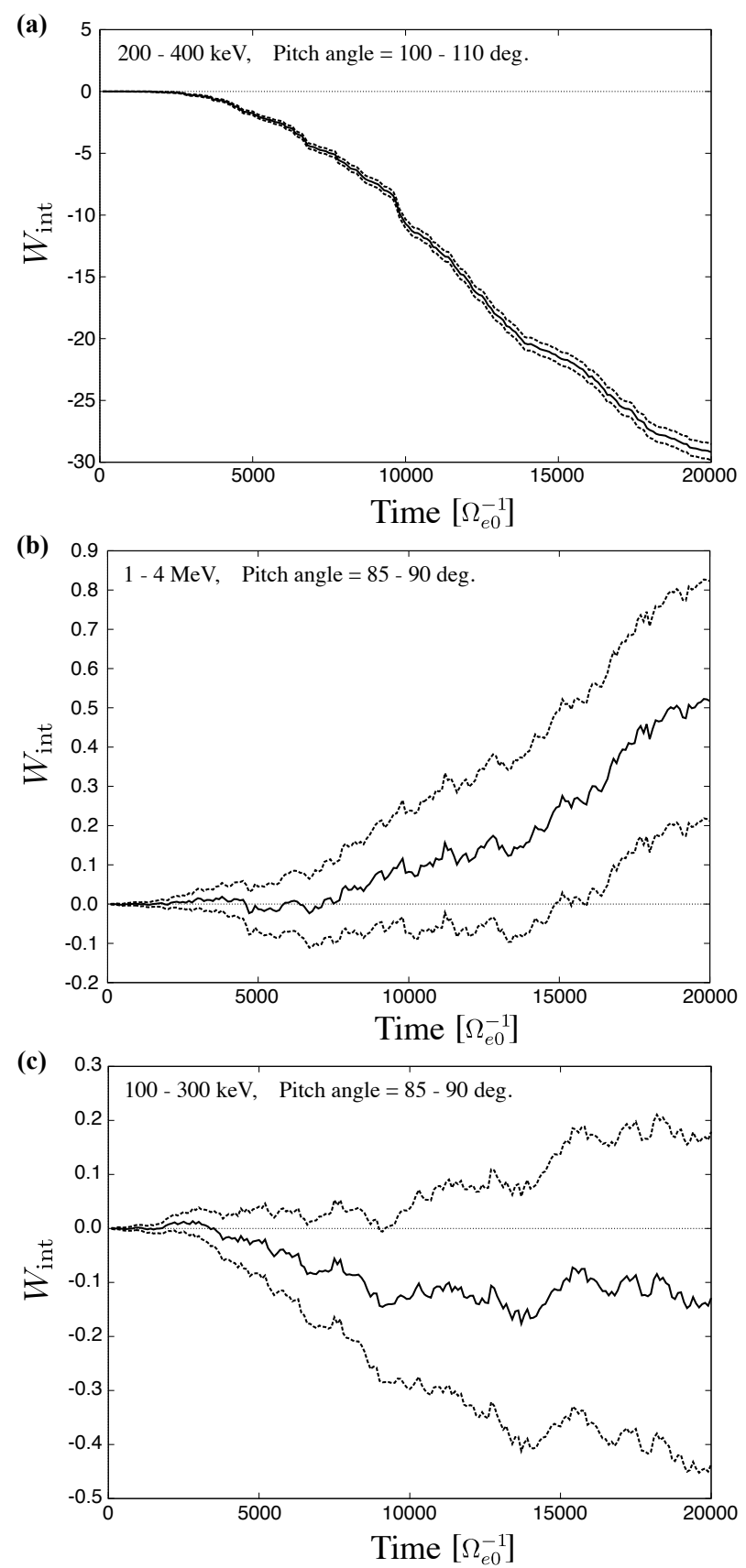

Fig. 4. Time histories of $W_{\text {int }}$ in (a) Region A, (b) Region B, and (c) Region $\mathrm{C}$ of velocity space. Energetic electrons passing through the observation point toward the magnetic equator are used in the computation. Dashed lines denote $W_{\text {int }} \pm 1.96 \sigma_{W}$.

$\sigma_{W}$, indicating that neither systematic wave-generation nor electron acceleration takes place in the Region C.

We expect that the enhancement of $W_{\text {int }}$ shown in Fig. $4 \mathrm{a}$ is a signature of the nonlinear wave growth of chorus elements. Based on the nonlinear growth theory (Omura et al., 2008), the explosive amplification of the wave fields can be explained by the contribution of nonlinear resonant currents
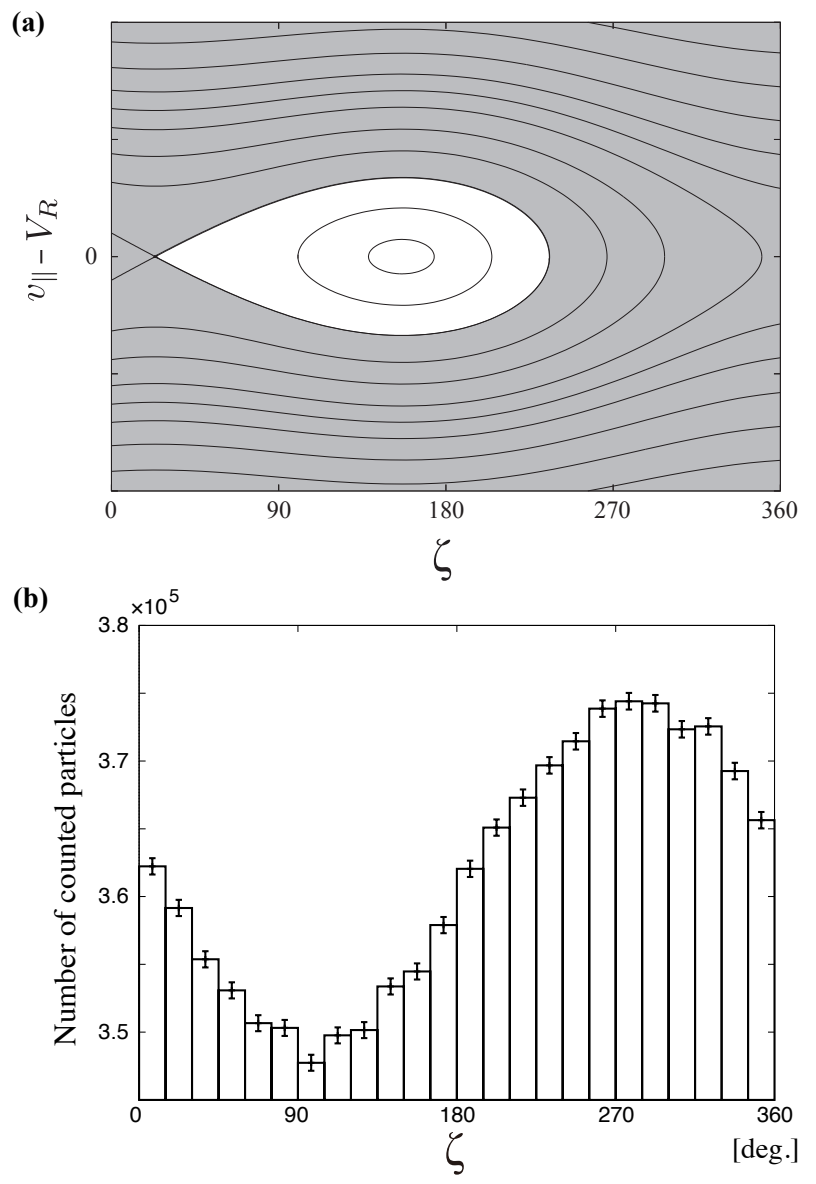

Fig. 5. (a) Trajectories of resonant electrons in $v_{\|}-\zeta$ phase space for the inhomogeneity factor $S=-0.4$. (b) The accumulated distribution in $\zeta$ space of energetic electrons in Region A. The estimated statistical noise $\sigma_{N}(\zeta)$ at each $\zeta$ bin is also shown.

arising from the nonlinear wave-particle interactions. The formation process of the nonlinear resonant currents has been explained in terms of the concentration of nonlinear trajectories of resonant electrons in the specific phase range of velocity phase space due to the presence of an electromagnetic electron hole. Since the optimum condition for the nonlinear wave growth of chorus elements is satisfied during the time interval of the simulation, an electromagnetic electron hole is formed in the $v_{\|}-\zeta$ space as shown in Fig. 5a corresponding to the condition $S=-0.4$, where $\zeta$ is the relative phase angle between the wave magnetic field $\boldsymbol{B}_{w}$ and $\boldsymbol{v}_{\perp}$ of an energetic electron, and $S$ is the inhomogeneity factor (Omura et al., 2008). Based on Fig. 5a, we expect a depletion of the distribution of energetic electrons in velocity phase space in the energy and pitch angle range showing large $W_{\text {int }}$ values. In the WPIA, the measurement of instantaneous wave and velocity vectors of electrons enables us to obtain the distribution of energetic electrons in wave phase space. Figure $5 b$ shows the accumulated distribution in $\zeta$ space of energetic electrons moving toward the magnetic equator with kinetic 

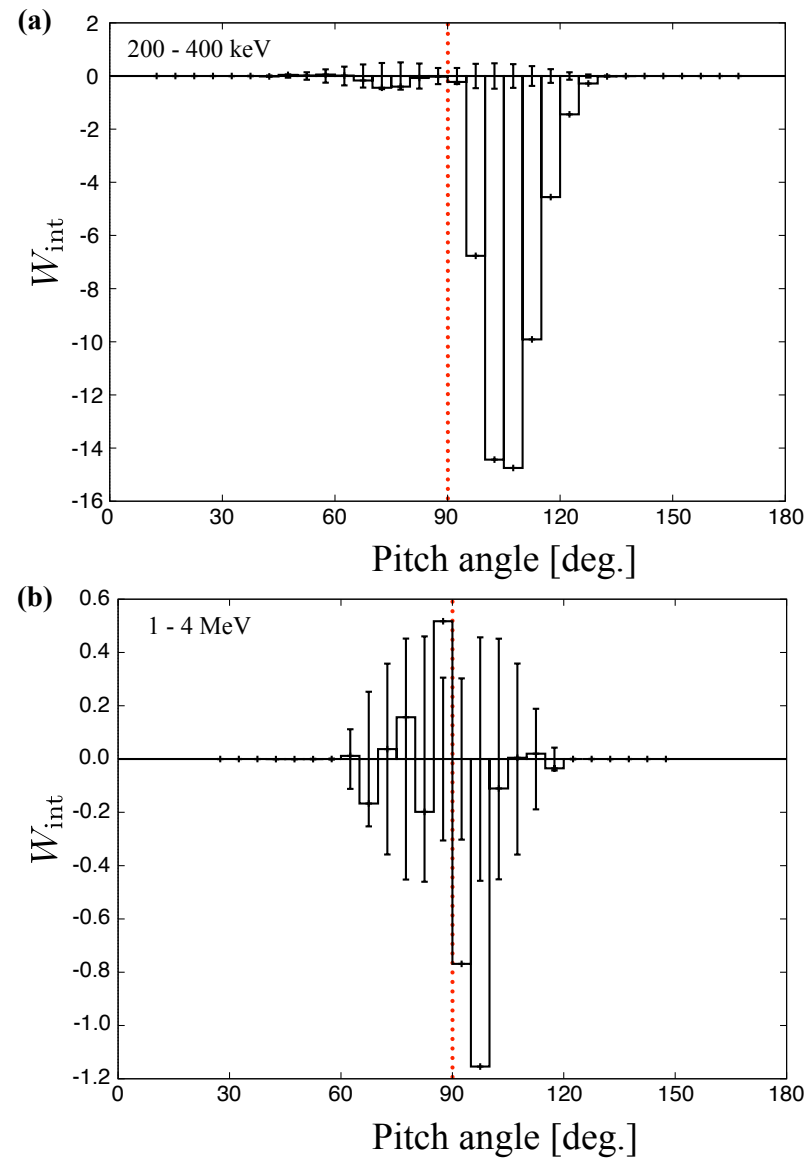

Fig. 6. $W_{\text {int }}$ computed (a) in the energy range from 200 to $400 \mathrm{keV}$ and (b) from 1 to $4 \mathrm{MeV}$ for the time interval from 0 to $20000 \Omega_{\mathrm{e} 0}^{-1}$ in pitch angle intervals of 5 degrees. The computed $1.96 \sigma_{W}$ of each pitch angle bin is also shown.

energies from 200 to $400 \mathrm{keV}$ and pitch angles from 100 to 110 degrees. We divided the wave phase angle into 24 bins, and the accumulation is conducted over the time interval from 0 to $20000 \Omega_{\mathrm{e} 0}^{-1}$, the same as that used in Fig. 3 . We find a clear depletion in the distribution in $\zeta$ space corresponding to the region where the electromagnetic electron hole is formed under the optimum condition for nonlinear wave growth. Assuming that the detection (count) of particles $N$ follows a Poisson distribution in each bin of the wave phase angle $\zeta$, the standard deviation $\sigma_{N}$ is equal to the square root of the particle counts (Knoll, 2000):

$\sigma_{N}(\zeta)=\sqrt{N(\zeta)}$

We show the estimated $\sigma_{N}(\zeta)$ as vertical bars in Fig. $5 \mathrm{~b}$ and find that the size of the depletion is statistically significant. This result clarifies that nonlinear resonant currents contributing to the large values of $W_{\text {int }}$ are maintained by the asymmetry of the electron distribution in velocity phase space due to the presence of the electromagnetic electron hole.

\section{Discussion}

We obtained a large $W_{\text {int }}$ with sufficient statistical significance to indicate wave generation in Region $\mathrm{A}$ and electron energisation in Region B, as shown in Fig. 4a and b. We also found fluctuations of $W_{\text {int }}$ in velocity phase space beyond just Regions A and B, as shown in Fig. 3. As an example, we found positive $W_{\text {int }}$ in the vicinity of Region $\mathrm{B}$, which represents energisation of relativistic electrons. An evaluation of the statistical significance of these $W_{\text {int }}$ values is shown in Fig. 6. Specifically, we show $W_{\text {int }}$ computed in the energy range from 200 to $400 \mathrm{keV}$ (Fig. 6a) and that from 1 to $4 \mathrm{MeV}$ (Fig. 6b) for the time interval from 0 to $20000 \Omega_{\mathrm{e} 0}^{-1}$ in pitch angle intervals of 5 degrees. The computed $1.96 \sigma_{W}$ of each pitch angle bin is also shown. According to the results shown in Fig. 6a, statistically significant $W_{\text {int }}$ representing wave generation were obtained not only Region A but pitch angles from 85 to 115 degrees. This result indicates that, in the energy range from 200 to $400 \mathrm{keV}$, energetic electrons of a wide range of pitch angles (from 85 to 115 degrees) contributed to the chorus generation, while energetic electrons in the pitch angle range from 100 to 110 degrees were particularly effective in the simulation. In Fig. $6 \mathrm{~b}$, a positive $W_{\text {int }}$ larger than $1.96 \sigma_{W}$ is observed only for the pitch angle range from 85 to 90 degrees, while a statistically significant negative $W_{\text {int }}$ was obtained in the pitch angle range from 90 to 100 degrees. Based on the consideration of the statistical significance of $W_{\text {int }}$, energy exchange between waves and particles is not evident in other pitch angle ranges.

In the electron hybrid code used in the present study, we assumed a large spatial inhomogeneity and neglected the parallel component of the electromagnetic wave field. These assumptions were necessary to reduce computational costs and are not critical for the comparison with theoretical studies; however, the necessary computation costs prevent us from considering realistic plasma parameter values, namely, those observed in the magnetosphere. In the simulation, we assumed energetic electrons having relatively higher kinetic energy and larger temperature anisotropy compared with typical observation results. As shown in Figs. 3 and 4, the energy range that mainly contributed to the wave growth in the simulation result was a few hundred $\mathrm{keV}$, while previous observations reported that the typical energy range of electrons for chorus generation is a few or tens of $\mathrm{keV}$ in the inner magnetosphere. As for a future study, it is necessary to carry out simulations under realistic initial conditions of the background magnetic field as well as velocity distribution function of energetic electrons and to conduct the WPIA in the results so as to evaluate feasibility of the WPIA quantitatively.

The quantity $W(t)$ actually has both parallel and perpendicular components with respect to the background magnetic field. Since the longitudinal component is neglected in the electron hybrid code, we only obtained the perpendicular components $W_{\perp}(t)=q \boldsymbol{E}_{\perp}(t) \cdot \boldsymbol{v}_{\perp}(t)$ in the pseudo-observations. Although the assumption of the 
parallel propagation of chorus is acceptable in the generation region close to the magnetic equator (e.g., Li et al., 2011), recent observations reported chorus propagating obliquely from the source region (Santolík et al., 2009; Chum et al., 2009). In addition, theoretical studies of the chorus generation process have suggested possible important roles for the parallel component of the wave electric field in understanding nonlinear wave damping at half the gyrofrequency (Omura et al., 2009). Interaction of energetic electrons with obliquely propagating whistler-mode waves is an important research target of the WPIA and, therefore, evaluation of $W_{\|}(t)$ is necessary in future study by simulation on solving the electromagnetic wave field in three dimensions.

\section{Summary}

By applying the WPIA to simulation results, we confirmed the effectiveness of the WPIA for studying the energy transfer process between relativistic electrons with whistler-mode chorus emissions. Using the simulation results, we computed $W(t)$, the inner product of the wave electric field observed at a fixed point and the velocity vector of each energetic electron passing through the fixed point along the field line, where $W(t)$ is the time variation of the kinetic energy of electrons interacting with plasma waves. By integrating $W(t)$ over time, we obtained $W_{\text {int }}$. We verified that large values of $W_{\text {int }}$, representing efficient wave-particle interactions, occurred in the simulation, which is consistent with previous studies (e.g., Katoh and Omura, 2007a; Omura et al., 2008, 2009; Katoh et al., 2008). We also derived an expression for the standard deviation $\sigma_{W}$ of $W_{\text {int }}$ and proposed that the statistical significance of the obtained $W_{\text {int }}$ be evaluated by comparing it with $\sigma_{W}$. We showed that statistically significant $W_{\text {int }}$ can be used to identify wave generation and acceleration of relativistic electrons. We also conducted an analysis to obtain the distribution of energetic electrons in $\zeta$ space and clarified that a deviation of the distribution in $\zeta$ space occurred in velocity phase space corresponding to the large $W_{\text {int }}$ values. This deviation in the $\zeta$ space distribution was consistent with the formation of nonlinear resonant currents proposed by recent theoretical studies on the generation mechanism of chorus emissions. The result of the present study demonstrates that the WPIA is useful for analysing the energy exchange between waves and particles and suggests that the statistical significance of the WPIA can be evaluated using the standard deviation $\sigma_{W}$ of $W_{\text {int }}$.

Acknowledgements. The computations described herein were performed using supercomputers at the Research Institute for Sustainable Humanosphere and Academic Center for Computing and Media Studies of Kyoto University, and the Solar-Terrestrial Environment Laboratory of Nagoya University. This work was supported by Grants-in-Aid for Scientific Research S (23224011) and for Young Scientists A (22684025) from the Ministry of Education, Science,
Sports and Culture of Japan. This work was supported by the Global COE program "Global Education and Research Center for Earth and Planetary Dynamics" of Tohoku University.

Topical Editor R. Nakamura thanks D. Nunn and one anonymous referee for their help in evaluating this paper.

\section{References}

Buckley, A. M., Gough, M. P., Alleyne, H., Yearby, K., and Willis, I.: Measurement of wave-particle interactions in the magnetosphere using the DWP particle correlator, Proceedings of ClusterII workshop, 303-306, 2000.

Chum, J., Santolík, O., Gurnett, D. A., and Pickett, J. S.: Oblique lower band chorus waves: Time shifts between discrete elements observed by the Cluster spacecraft, J. Geophys. Res., 114, A00F02, doi:10.1029/2009JA014366, 2009.

Ebihara, Y. and Miyoshi, Y.: Dynamic inner magnetosphere: A tutorial and recent advances, in: The Dynamic Magnetosphere, IAGA Special Sopron Book Series 3, edited by: Liu, W. and Fujimoto, M., pp. 145-187, Springer Science+Business Media B.V., doi:10.1007/978-94-007-0501-2_9, 2011.

Ergun, R. E., Carlson, C. W., McFadden, J. P., Clemmons, J. H., and Boehm, M. H.: Langmuir wave growth and electron bunching: Results from a wave-particle correlator, J. Geophys. Res., 96, 225-238, 1991.

Ergun, R. E., McFadden, J. P., and Carlson, C. W.: Wave-particle correlator instrument design, in: Measurement Techniques in Space Plasmas: Particles, AGU, Geophysical Monograph, 102, 325-331, 1998.

Fukuhara, H., Kojima, H., Ueda, Y., Omura, Y., Katoh, Y., and Yamanaka, H.: A new instrument for the study of wave-particle interactions in space: One-chip Wave-Particle Interaction Analyzer, Earth Planets Space, 61, 765-778, 2009.

Furuya, N., Omura, Y., and Summers, D.: Relativistic turning acceleration of radiation belt electrons by whistler mode chorus, J. Geophys. Res., 113, A04224, doi:10.1029/2007JA012478, 2008.

Gough, M. P., Hardy, D. A., Oberhardt, M. R., Burke, W. J., Gentile, L. C., McNeil, B., Bounar, K., Thompson, D. C., and Raitt, W. J.: Correlator measurements of megahertz wave-particle interactions during electron beam operations on STS, J. Geophys. Res., 100, 21561-21575, 1995.

Hikishima, M., Yagitani, S., Omura, Y., and Nagano, I.: Full particle simulation of whistler-mode rising chorus emissions in the magnetosphere, J. Geophys. Res., 114, A01203, doi:10.1029/2008JA013625, 2009.

Horne, R. B., Meredith, N. P., Thorne, R. M., Heynderickx, D., Iles, R. H. A., and Anderson, R. R.: Evolution of energetic electron pitch angle distributions during storm time electron acceleration to megaelectronvolt energies, J. Geophys. Res., 108, 1016, doi:10.1029/2001JA009165, 2003.

Kasahara, Y., Miyoshi, Y., Omura, Y., Verkhoglyadova, O. P., Nagano, I., Kimura, I., and Tsurutani, B. T.: Simultaneous satellite observations of VLF chorus, hot and relativistic electrons in a magnetic storm "recovery" phase, Geophys. Res. Lett., 36, L01106, doi:10.1029/2008GL036454, 2009.

Katoh, Y. and Omura, Y.: Acceleration of relativistic electrons due to resonant scattering by whistler mode waves generated by temperature anisotropy in the inner magnetosphere, J. Geophys. Res., 109, A12214, doi:10.1029/2004JA010654, 2004. 
Katoh, Y. and Omura, Y.: A study of generation mechanism of VLF triggered emission by self-consistent particle code, J. Geophys. Res., 111, A12207, doi:10.1029/2006JA011704, 2006.

Katoh, Y. and Omura, Y.: Computer simulation of chorus wave generation in the Earth's inner magnetosphere, Geophys. Res. Lett., 34, L03102, doi:10.1029/2006GL028594, 2007a.

Katoh, Y. and Omura, Y.: Relativistic particle acceleration in the process of whistler-mode chorus wave generation, Geophys. Res. Lett., 34, L13102, doi:10.1029/2007GL029758, 2007b.

Katoh, Y. and Omura, Y.: Amplitude dependence of frequency sweep rates of whistler-mode chorus emissions, J. Geophys. Res., 116, A07201, doi:10.1029/2011JA016496, 2011.

Katoh, Y., Omura, Y., and Summers, D.: Rapid energization of radiation belt electrons by nonlinear wave trapping, Ann. Geophys., 26, 3451-3456, doi:10.5194/angeo-26-3451-2008, 2008.

Knoll, G. F.: Radiation Detection and Measurement, 3rd ed., Wiley, New York, 2000.

Li, W., Bortnik, J., Thorne, R. M., and Angelopoulos, V.: Global distribution of wave amplitudes and wave normal angles of chorus waves using THEMIS wave observations, J. Geophys. Res., 116, A12205, doi:10.1029/2011JA017035, 2011.

Matsumoto, H.: Coherent nonlinear effects on electromagnetic wave-particle interactions, Space Sci. Rev., 42, 429-448, 1985.

Miyoshi, Y., Morioka, A., Obara, T., Misawa, H., Nagai, T., and Kasahara, Y.: Rebuilding process of the outer radiation belt during the 3 November 1993 magnetic storm: NOAA and EXOS-D observations, J. Geophys. Res., 108, 1004, doi:10.1029/2001JA007542, 2003.

Miyoshi, Y., Morioka, A., Kataoka, R., Kasahara, Y., and Mukai, T.: Evolution of the outer radiation belt during the November 1993 storms driven by corotating interaction regions, J. Geophys. Res., 112, A05210, doi:10.1029/2006JA012148, 2007.

Miyoshi, Y., Ono, T., Takashima, T., Asamura, K., Hirahara, M., Kasaba, Y., Matsuoka, A., Kojima, H., Shiokawa, K., Seki, K., Fujimoto, M., Nagatsuma, T., Cheng, C. Z., Kazama, Y., Kasahara, S., Matsumoto, H., Higashio, N., Kumamoto, A., Yagitani, S., Kasahara, Y., Ishisaka, K., Katoh, Y., Ebihara, Y., Omura, Y., Nose, M., Hori, T., Miyashita, Y., Tanaka, Y., Segawa, T., and ERG-working group: The Energization and Radiation in Geospace (ERG) Project, in: Dynamics of the Earth's Radiation Belts and Inner Magnetosphere, Geophys. Monogr. Ser., 199, edited by: Summers, D., Mann, I. R., Baker, D. N., and Schulz, M., pp. 103-116, AGU, Washington, D.C., doi:10.1029/2012GM001304, 2012.

Nunn, D.: A self-consistent theory of triggered VLF emissions, Planet. Space Sci., 22, 349-378, 1974.

Nunn, D. and Omura, Y.: A computational and theoretical analysis of falling frequency VLF emissions, J. Geophys. Res., 117, A08228, doi:10.1029/2012JA017557, 2012.
Nunn, D., Omura, Y., Matsumoto, H., Nagano, I., and Yagitani, S.: The numerical simulation of VLF chorus and discrete emissions observed on the Geotail satellite using a Vlasov code, J. Geophys. Res., 102, 27083-27097, 1997.

Omura, Y. and Nunn, D.: Triggering process of whistler mode chorus emissions in the magnetosphere, J. Geophys. Res., 116, A05205, doi:10.1029/2010JA016280, 2011.

Omura, Y., Furuya, N., and Summers, D.: Relativistic turning acceleration of resonant electrons by coherent whistler mode waves in a dipole magnetic field, J. Geophys. Res., 111, A06236, doi:10.1029/2006JA012243, 2007.

Omura, Y., Katoh, Y., and Summers, D.: Theory and simulation of the generation of whistler-mode chorus, J. Geophys. Res., 113, A04223, doi:10.1029/2007JA012622, 2008.

Omura, Y., Hikishima, M., Katoh, Y., Summers, D., and Yagitani, S.: Nonlinear mechanisms of lower-band and upper-band VLF chorus emissions in the inner magnetosphere, J. Geophys. Res., 114, A07217, doi:10.1029/2009JA014206, 2009.

Santolík, O., Gurnett, D. A., Pickett, J. S., Chum, J., and CornilleauWehrlin, N.: Oblique propagation of whistler mode waves in the chorus source region, J. Geophys. Res., 114, A00F03, doi:10.1029/2009JA014586, 2009.

Shprits, Y., Dmitriy, D. A., Meredith, N. P., and Elkington, S. R.: Review of modeling of losses and sources of relativistic electrons in the outer radiation belt II: Local acceleration and loss, J. Atmos. Solar-Terr. Phys., 70, 1694-1713, 2008.

Summers, D. and Omura, Y.: Ultra-relativistic acceleration of electrons in planetary magnetospheres, Geophys. Res. Lett., 34, L24205, doi:10.1029/2007GL032226, 2007.

Summers, D., Thorne, R. M., and Xiao, F.: Relativistic theory of wave-particle resonant diffusion with application to electron acceleration in the magnetosphere, J. Geophys. Res., 103, 2048720500, 1998.

Thorne, R. M.: Radiation belt dynamics: The importance of wave-particle interactions, Geophys. Res. Lett., 37, L22107, doi:10.1029/2010GL044990, 2010.

Trakhtengerts, V. Y.: Magnetosphere cyclotron maser: backward wave oscillator generation regime, J. Geophys. Res., 100, 1720517210, 1995.

Trakhtengerts, V. Y.: A generation mechanism for chorus emission, Ann. Geophys., 17, 95-100, doi:10.1007/s00585-999-0095-4, 1999.

Xiao, F., Thorne, R. M., and Summers, D.: Instability of electromagnetic R-mode waves in a relativistic plasma, Phys. Plasmas, 5, 2489-2497, 1998. 